\title{
Collision of an innermost stable circular orbit particle around a Kerr black hole
}

\author{
${ }^{1}$ Tomohiro Harada* and ${ }^{2}$ Masashi Kimurat \\ ${ }^{1}$ Department of Physics, Rikkyo University, Toshima, Tokyo 175-8501, Japan \\ ${ }^{2}$ Department of Mathematics and Physics, Graduate School of Science, \\ Osaka City University, Osaka 558-8585, Japan
}

(Dated: September 19, 2018)

\begin{abstract}
We derive a general formula for the center-of-mass (CM) energy for the near-horizon collision of two particles of the same rest mass on the equatorial plane around a Kerr black hole. We then apply this formula to a particle which plunges from the innermost stable circular orbit (ISCO) and collides with another particle near the horizon. It is found that the maximum value of the CM energy $E_{\mathrm{cm}}$ is given by $E_{\mathrm{cm}} /\left(2 m_{0}\right) \simeq 1.40 / \sqrt[4]{1-a_{*}^{2}}$ for a nearly maximally rotating black hole, where $m_{0}$ is the rest mass of each particle and $a_{*}$ is the nondimensional Kerr parameter. This coincides with the known upper bound for a particle which begins at rest at infinity within a factor of 2. Moreover, we also consider the collision of a particle orbiting the ISCO with another particle on the ISCO and find that the maximum CM energy is then given by $E_{\mathrm{cm}} /\left(2 m_{0}\right) \simeq 1.77 / \sqrt[6]{1-a_{*}^{2}}$. In view of the astrophysical significance of the ISCO, this result implies that particles can collide around a rotating black hole with an arbitrarily high CM energy without any artificial fine-tuning in an astrophysical context if we can take the maximal limit of the black hole spin or $a_{*} \rightarrow 1$. On the other hand, even if we take Thorne's bound on the spin parameter into account, highly or moderately relativistic collisions are expected to occur quite naturally, for $E_{\mathrm{cm}} /\left(2 m_{0}\right)$ takes 6.95 (maximum) and 3.86 (generic) near the horizon and 4.11 (maximum) and 2.43 (generic) on the ISCO for $a_{*}=0.998$. This implies that high-velocity collisions of compact objects are naturally expected around a rapidly rotating supermassive black hole. Implications to accretion flows onto a rapidly rotating black hole are also discussed.
\end{abstract}

PACS numbers: 04.70.-s, 04.70.Bw, 97.60.Lf

*Electronic address: harada@rikkyo.ac.jp 
†Electronic address: mkimura@sci.osaka-cu.ac.jp 


\section{INTRODUCTION}

Recently, Banados, Silk and West [1] showed that if two particles which begin at rest at infinity collide near the horizon of a maximally rotating Kerr black hole [2] and if the angular momentum of either particle is fine-tuned, the center-of-mass (CM) energy $E_{\mathrm{cm}}$ of the two particles can be arbitrarily high and hence the maximally rotating black hole might be regarded as a Planck-energy-scale collider. We here call this the Banados-SilkWest (BSW) effect. This scenario was subsequently criticized [3, 4] from several points, such as astrophysical bounds on the black hole spin parameter, the effects of gravitational waves, the self-gravity of the particles and the long proper time needed for such a collision. In the near-maximal rotation, the CM energy of two particles of mass $m_{0}$ is bounded by $E_{\mathrm{cm}} /\left(2 m_{0}\right) \sim 2.41 / \sqrt[4]{1-a_{*}^{2}}$, where $a_{*}$ is the nondimensional Kerr parameter [3], 4]. On the other hand, Grib and Pavlov [5] proposed a slightly different mechanism that $E_{\mathrm{cm}}$ can be arbitrarily high even in the non-maximal rotation if the particle has experienced multiple scattering and fine-tuned its angular momentum before the relevant collision. The BSW

effect is also analyzed in the Kerr-Newman family of black holes [6], general stationary and axisymmetric black holes [7], and static charged black holes [8].

In the BSW effect, to obtain such an arbitrarily high $E_{\mathrm{cm}}$, the angular momentum of either particle must be fine-tuned. However, there is a natural mechanism in astrophysics to tune the particle's energy and angular momentum. This is the innermost stable circular orbit (ISCO). The ISCO around a Kerr black hole is studied in detail by Bardeen, Press and Teukolsky [9].

In the geometrically thin and optically thick accretion disk model [10, 11], which is known as the standard accretion disk model, a rotating fluid or plasma gradually takes a circular orbit which is closer to the black hole as the fluid transfers its angular momentum outwards and releases its energy by electromagnetic radiation in the time scale of viscosity, which is much longer than the black hole dynamical time scale. This electromagnetic emission can be observed by radio interferometers and X-ray observation satellites. Once the fluid reaches the inner edge of the accretion disk, which is given by the ISCO, it begins to plunge into the black hole in the dynamical time scale [26]. In the plunging phase, its energy and angular momentum are approximately conserved. It should be noted that due to the accretion of radiation emitted from the disk, there is an astrophysical upper bound on the 
nondimensional Kerr parameter, what we call Thorne's bound, $\left|a_{*}\right| \lesssim 0.998$ [14].

Another important example where the ISCO plays a crucial role is inspirals of stellarmass compact objects into supermassive black holes, which are called extreme mass-ratio inspirals. Extreme mass-ratio inspirals are interesting sources of gravitational waves for the Laser Interferometric Space Antenna [15]. If instead the central mass is an intermediate-mass black hole, these are interesting sources for the DECi-hertz Interferometer Gravitational wave Observatory [16, 17] and the Big Bang Observatory [18, 19]. Also in this case, an inspiralling compact object gradually takes a circular orbit which is closer to the black hole as the object transfers its angular momentum outwards and releases its energy by gravitational waves in the time scale of gravitational radiation, which is much longer than the dynamical time scale. Once the compact object reaches the ISCO, it begins to plunge into the black hole in the dynamical time scale. In the plunging phase, its energy and angular momentum are approximately conserved.

In this paper, we derive a general formula for the CM energy for the near-horizon collision of two particles on the equatorial plane around a Kerr black hole, which is valid in both the maximal and non-maximal rotation cases. Then, we apply this formula to the nearhorizon collision of two particles, either of which is plunging from the ISCO. We find that the BSW effect occurs in the near-maximal rotation and that the maximum value for the CM energy of the ISCO particles is the same as the upper bound for the particles initially at rest at infinity within a factor of 2 . This implies that the BSW effect is not an artificial but physically realistic astrophysical phenomenon. We also consider the collision of a particle orbiting the ISCO with another generic particle on the ISCO and find that the associated CM energy can also be arbitrarily high in exactly the same sense as BSW's, although the dependence on the black hole spin parameter is quite different. We neglect the effects of gravitational waves and the self-gravity of the particles.

This paper is organized as follows. In Sec. II we briefly review particle orbits and the CM energy for the collision of two particles in the Kerr spacetime. In Sec. III, we discuss particle orbits near the horizon and derive a general formula for the CM energy for the near-horizon particle collision. In Sec. IV, we apply this formula to a particle which plunges from the ISCO and obtain the CM energy for different collisions. In Sec. V, we investigate the collision of a particle orbiting the ISCO with another particle on the ISCO. Section VI is devoted to conclusion and discussion. We use the units in which $c=G=1$ and the abstract 
index notation of Wald [20].

\section{CM ENERGY FOR PARTICLE COLLISION IN THE KERR SPACETIME}

In this section, we briefly review particle orbits and the CM energy for the two-particle collision on the equatorial plane of the Kerr spacetime in the general situation, following [1, 4, 5]. We use a similar notation to that of Grib and Pavlov [5].

\section{A. Particle orbits in the Kerr spacetime}

The line element in the Kerr spacetime in the Boyer-Lindquist coordinates is given by [2, 20, 21]

$$
\begin{aligned}
d s^{2}= & -\left(1-\frac{2 M r}{\rho^{2}}\right) d t^{2}-\frac{4 M a r \sin ^{2} \theta}{\rho^{2}} d \phi d t+\frac{\rho^{2}}{\Delta} d r^{2}+\rho^{2} d \theta^{2} \\
& +\left(r^{2}+a^{2}+\frac{2 M r a^{2} \sin ^{2} \theta}{\rho^{2}}\right) \sin ^{2} \theta d \phi^{2}
\end{aligned}
$$

where $a$ and $M$ are, respectively, the spin and mass parameters, $\rho^{2}=r^{2}+a^{2} \cos ^{2} \theta$ and $\Delta=r^{2}-2 M r+a^{2}$. We assume $a \geq 0$ without loss of generality. If $a^{2} \leq M^{2}, \Delta$ vanishes at $r=r_{ \pm}=M \pm \sqrt{M^{2}-a^{2}}$, where $r=r_{+}$and $r=r_{-}$correspond to an event horizon and a Cauchy horizon, respectively. Here, we denote $r_{+}=r_{H}$ and $r_{-}=r_{C}$. In this coordinate system, the time translational and the axial Killing vectors are respectively given by

$$
\xi^{a}=\left(\frac{\partial}{\partial t}\right)^{a}, \quad \psi^{a}=\left(\frac{\partial}{\partial \phi}\right)^{a} .
$$

The surface gravity of the Kerr black hole is given by

$$
\kappa=\frac{\sqrt{M^{2}-a^{2}}}{r_{H}^{2}+a^{2}} .
$$

Thus, the black hole has a vanishing surface gravity and hence is extremal for the maximal rotation $a^{2}=M^{2}$, while it is subextremal for the non-maximal rotation $a^{2}<M^{2}$. The angular velocity of the horizon is given by

$$
\Omega_{H}=\frac{a}{r_{H}^{2}+a^{2}}=\frac{a}{2 M\left(M+\sqrt{M^{2}-a^{2}}\right)} .
$$

The Killing vector $\chi^{a}=\xi^{a}+\Omega_{H} \psi^{a}$ is a null generator of the event horizon. 
Here we concentrate on particles which move on the equatorial plane $\theta=\pi / 2$. Then, the four velocity $u^{a}=\dot{x}^{a}$ of the particle has a vanishing $\theta$ component, i.e., $u^{\theta}=0$, where the dot denotes the differentiation with respect to the affine parameter of the geodesic. From Eq. (2.1), the line element on the equatorial plane in the Kerr spacetime is given by

$$
d s^{2}=-\left(1-\frac{2 M}{r}\right) d t^{2}-\frac{4 a M}{r} d t d \phi+\frac{r^{2}}{\Delta} d r^{2}+\left(r^{2}+a^{2}+\frac{2 M a^{2}}{r}\right) d \phi^{2} .
$$

Associated with the Killing vectors $\xi^{a}$ and $\psi^{a}$, we have the following conserved quantities along a geodesic on the equatorial plane:

$$
\begin{aligned}
& e=-g_{a b} \xi^{a} u^{b}=-u_{t}=-\left(g_{t t} u^{t}+g_{t \phi} u^{\phi}\right), \\
& L=g_{a b} \psi^{a} u^{b}=u_{\phi}=g_{\phi t} u^{t}+g_{\phi \phi} u^{\phi}
\end{aligned}
$$

where $e$ and $L$ correspond the specific energy and angular momentum, respectively. Solving the above for $u^{t}$ and $u^{\phi}$, we have

$$
\begin{aligned}
& \dot{t}=\frac{1}{\Delta}\left[\left(r^{2}+a^{2}+\frac{2 M a^{2}}{r}\right) e-\frac{2 M a}{r} L\right], \\
& \dot{\phi}=\frac{1}{\Delta}\left[\left(1-\frac{2 M}{r}\right) L+\frac{2 M a}{r} e\right] .
\end{aligned}
$$

To have $\dot{t} \geq 0$, the condition

$$
\left(r^{2}+a^{2}+\frac{2 M a^{2}}{r}\right) e-\frac{2 M a}{r} L \geq 0
$$

must be satisfied outside the event horizon. In the limit to the horizon $r \rightarrow r_{H}$ from outside, this condition reduces to

$$
l \leq l_{H}=\frac{2\left(1+\sqrt{1-a_{*}^{2}}\right)}{a_{*}} e=\frac{e}{M \Omega_{H}}
$$

or

$$
e \geq \Omega_{H} L
$$

where we put $a_{*}=a / M$ and $l=L / M$. In terms of $a_{*}, 0 \leq a_{*}<1$ for the subextremal case, while $a_{*}=1$ for the extremal case.

Substituting Eqs. (2.4) and (2.5) into the normalization condition $u^{a} u_{a}=-1$ with $\theta=$ $\pi / 2$ and $u^{\theta}=0$, or

$$
-\left(1-\frac{2 M}{r}\right)\left(u^{t}\right)^{2}-\frac{4 a M}{r} u^{t} u^{\phi}+\frac{r^{2}}{\Delta}\left(u^{r}\right)^{2}+\left(r^{2}+a^{2}+\frac{2 M a^{2}}{r}\right)\left(u^{\phi}\right)^{2}=-1,
$$


we obtain

$$
\frac{1}{2} \dot{r}^{2}+V_{\text {eff }}(r)=0
$$

where the effective potential $V_{\text {eff }}(r)$ is given by

$$
V_{\mathrm{eff}}(r)=-\frac{M}{r}+\frac{L^{2}-a^{2}\left(e^{2}-1\right)}{2 r^{2}}-\frac{M(L-a e)^{2}}{r^{3}}-\frac{e^{2}-1}{2} .
$$

The effective potential can be efficiently analyzed by introducing $y=M / r$. We put

$$
g(y)=-2\left(l-a_{*} e\right)^{2} y^{3}+\left[l^{2}-a_{*}^{2}\left(e^{2}-1\right)\right] y^{2}-2 y-\left(e^{2}-1\right)
$$

and

$$
D(y)=a_{*}^{2} y^{2}-2 y+1
$$

Then, we have $V_{\text {eff }}=g(y) / 2$ and $\Delta=r^{2} D(y)$. There are two positive roots of $D=0$ for $0<a_{*} \leq 1$, which are given by

$$
y_{H}=\frac{1-\sqrt{1-a_{*}^{2}}}{a_{*}^{2}}, \quad y_{C}=\frac{1+\sqrt{1-a_{*}^{2}}}{a_{*}^{2}},
$$

where $y_{H}=M / r_{H}$ and $y_{C}=M / r_{C}$ correspond to the event horizon and the Cauchy horizon, respectively. These two roots coincide with each other at $y=1$ in the extremal case $a_{*}=1$. The region outside the horizon is transformed into $0<y<y_{H}$. We should note the following useful relation

$$
\Omega_{H}=\frac{a_{*} y_{H}}{2 M} .
$$

For a particle which is initially at rest at infinity, i.e., marginally bound $e=1$, to reach the horizon, the potential $g(y)$ must be nonpositive for $0<y<y_{H}$. For $e=1$, the potential is given by

$$
g(y)=-y\left[2\left(l-a_{*}\right)^{2} y^{2}-l^{2} y+2\right] .
$$

Thus, the condition reduces to that $2\left(l-a_{*}\right)^{2} y^{2}-l^{2} y+2$ is nonnegative. After some straightforward calculation, we can obtain the following condition [5]

$$
-2\left(1+\sqrt{1+a_{*}}\right)=l_{L} \leq l \leq l_{R}=2\left(1+\sqrt{1-a_{*}}\right) .
$$

A similar condition also exists for the non-marginally bound case. However, we should note that this does not apply if the particle scatters with other particles and changes its energy and angular momentum on the way to the horizon. 


\section{B. CM energy of two particles in the Kerr spacetime}

We consider the collision of two particles 1 and 2 of the same rest mass $m_{0}$. We assume that the two particles are at the same spacetime point. The four momentum of particle $i$ $(i=1,2)$ is given by

$$
p_{i}^{a}=m_{0} u_{i}^{a},
$$

where $u_{i}^{a}$ is the four velocity of particle $i$. The sum of the two momenta is given by

$$
p_{\mathrm{t}}^{a}=p_{1}^{a}+p_{2}^{a} .
$$

The CM energy $E_{\mathrm{cm}}$ of the two particles is then given by

$$
E_{\mathrm{cm}}^{2}=-p_{\mathrm{t}}^{a} p_{\mathrm{t} a}=2 m_{0}^{2}\left(1-g_{a b} u_{1}^{a} u_{2}^{b}\right)
$$

On the background metric (2.1), using Eqs. (2.4), (2.5), (2.6) and (2.8) in Eq. (2.9), the $\mathrm{CM}$ energy of two particles 1 and 2 in the Kerr spacetime is calculated as

$$
\begin{aligned}
\frac{E_{\mathrm{cm}}^{2}}{2 m_{0}^{2}} & =1-g_{t t} u_{1}^{t} u_{2}^{t}-g_{t \phi}\left(u_{1}^{t} u_{2}^{\phi}+u_{1}^{\phi} u_{2}^{t}\right)-g_{r r} u_{1}^{r} u_{2}^{r}-g_{\phi \phi} u_{1}^{\phi} u_{2}^{\phi} \\
& =1-e_{1} e_{2}+\frac{F(y)-G(y)}{D(y)}
\end{aligned}
$$

where $e_{i}$ and $l_{i}$ are $e$ and $l$ for particle $i$,

$$
\begin{aligned}
F(y) & =2\left[a_{*}^{2} y^{2}(1+y)+(1-y)\right] e_{1} e_{2}-2 a_{*} y^{3}\left(e_{1} l_{2}+l_{1} e_{2}\right)-(1-2 y) y^{2} l_{1} l_{2}, \\
G & =\left( \pm \sqrt{-g_{1}}\right)\left( \pm \sqrt{-g_{2}}\right), \\
g_{i}(y) & =-2\left(l_{i}-a_{*} e_{i}\right)^{2} y^{3}+\left[l_{i}^{2}-a_{*}^{2}\left(e_{i}^{2}-1\right)\right] y^{2}-2 y-\left(e_{i}^{2}-1\right), \\
D(y) & =a_{*}^{2} y^{2}-2 y+1
\end{aligned}
$$

and the sign in front of $\sqrt{-g_{i}}$ in the expression of $G$ in Eq. (2.12) corresponds to the sign of $u_{i}^{r}$. In the following we assume $\dot{r} \leq 0$ for both particles and hence $G(y)=\sqrt{\left(-g_{1}\right)\left(-g_{2}\right)}$.

\section{NEAR-HORIZON COLLISION AROUND A KERR BLACK HOLE}

\section{A. Near-horizon collision around a subextremal Kerr black hole}

We will see the near-horizon behavior of particles with the angular momentum $l=l_{H}$, which we call critical, and smaller angular momentum $l<l_{H}$, which we call subcritical. We 
find

$$
g\left(y_{H}\right)=-y_{H}^{2}\left(2 e-a_{*} y_{H} l\right)^{2}=-a_{*}^{2} y_{H}^{4}\left(l_{H}-l\right)^{2},
$$

noting that $l_{H}$ can be written in terms of $a_{*}, y_{H}$ and $e$ as $l_{H}=2 e /\left(a_{*} y_{H}\right)$. Hence, $g\left(y_{H}\right) \leq 0$. It is interesting to see whether a particle with $l=l_{H}$ which approaches the horizon is possible. With $l=l_{H}$, we have $g\left(y_{H}\right)=0$, while

$$
g^{\prime}\left(y_{H}\right)=-2 \frac{\sqrt{1-a_{*}^{2}}}{a_{*}^{2}}\left[\left(1+\sqrt{1-a_{*}^{2}}\right)^{2} e^{2}+a_{*}^{2}\right],
$$

where the prime denotes the differentiation with respect to the argument. This is negative for the subextremal Kerr case $a_{*}^{2}<1$. This means that for the subextremal case, the effective potential $g(y)$ is positive in the vicinity of the horizon and hence a particle with the angular momentum $l=l_{H}$ is prohibited to approach the horizon. On the other hand, there does exist a particle with slightly smaller angular momentum $l=l_{H}-\delta$ which approaches the horizon in the vicinity of the horizon. The CM energy for the collision involving this particle can be arbitrarily high in the limit $\delta \rightarrow 0$ even in the subextremal Kerr case [5]. For the subcritical orbit $l<l_{H}$, Eq. (3.1) implies that $r$ is given near the horizon in terms of the particle's proper time $\tau$ as

$$
r-r_{H} \simeq-a_{*} y_{H}^{2}\left(l_{H}-l\right) \tau+\text { const. }
$$

This means that for the fixed initial radius, a subcritical particle reaches the horizon after a proper time inversely proportional to $\left(l_{H}-l\right)$.

Then, we will take the limit to the horizon in Eq. (2.10) to consider the collision near the horizon. Noting

$$
F\left(y_{H}\right)=a_{*}^{2} y_{H}^{4}\left(l_{H 1}-l_{1}\right)\left(l_{H 2}-l_{2}\right)
$$

where $l_{H i}$ is the critical angular momentum $l_{H}$ for particle $i(i=1,2)$, combined with Eq. (3.1), we can see that the terms of $O(1)$ in the numerator $F-G$ of the fraction on the right-hand side of Eq. (2.10) cancel out. The nonvanishing contribution comes from the next order terms. Using l'Hospital's rule, the result is the following:

$$
\frac{E_{\mathrm{cm}}^{2}}{2 m_{0}^{2}}=1-e_{1} e_{2}+\lim _{y \rightarrow y_{H}} \frac{F^{\prime}-G^{\prime}}{D^{\prime}} .
$$


The derivatives are given by

$$
\begin{aligned}
F^{\prime}(y) & =2\left[a_{*}^{2}\left(2 y+3 y^{2}\right)-1\right] e_{1} e_{2}-6 a_{*} y^{2}\left(e_{1} l_{2}+l_{1} e_{2}\right)-2 y(1-3 y) l_{1} l_{2}, \\
g_{i}^{\prime}(y) & =-6\left(l_{i}-a_{*} e_{i}\right)^{2} y^{2}+2\left[l_{i}^{2}-a_{*}^{2}\left(e_{i}^{2}-1\right)\right] y-2, \\
D^{\prime}(y) & =2\left(a_{*}^{2} y-1\right), \\
G^{\prime} & =G \frac{1}{2}\left(\frac{g_{1}^{\prime}}{g_{1}}+\frac{g_{2}^{\prime}}{g_{2}}\right) .
\end{aligned}
$$

From this form, we can see that there are two first-order poles, where $g_{i}\left(y_{H}\right)=0$ for $i=1,2$. By implementing the calculation and taking the limit, we reach the following formula:

$$
\frac{E_{\mathrm{cm}}}{2 m_{0}}=\sqrt{1+\frac{4\left[\left(l_{H 1}-l_{1}\right)-\left(l_{H 2}-l_{2}\right)\right]^{2}+\left(l_{H 1} l_{2}-l_{H 2} l_{1}\right)^{2}}{16\left(l_{H 1}-l_{1}\right)\left(l_{H 2}-l_{2}\right)}} .
$$

This is the formula for the CM energy of two particles along the general geodesic orbits on the equatorial plane. We should note that the right-hand side is given only in terms of the particles' angular momenta $l_{1}$ and $l_{2}$ and their critical values $l_{H 1}$ and $l_{H 2}$. In terms of the quantities which have more direct physical meanings, Eq. (3.4) can be rewritten as follows:

$$
\frac{E_{\mathrm{cm}}}{2 m_{0}}=\sqrt{1+\frac{4 M^{2}\left[\left(e_{1}-\Omega_{H} L_{1}\right)-\left(e_{2}-\Omega_{H} L_{2}\right)\right]^{2}+\left(e_{1} L_{2}-e_{2} L_{1}\right)^{2}}{16 M^{2}\left(e_{1}-\Omega_{H} L_{1}\right)\left(e_{2}-\Omega_{H} L_{2}\right)}},
$$

where $L_{i}$ is $L$ for particle $i$. In fact, as we will prove in Sec. IIIB, Eq. (3.4) or equivalently Eq. (3.5) is valid even for the extremal Kerr black hole simply by taking the near-extremal limit $a_{*} \rightarrow 1$. The necessary condition for obtaining an arbitrarily high $E_{\mathrm{cm}}$ is therefore $l \rightarrow l_{H}$ or $\Omega_{H} L \rightarrow e$ for either of the two particles.

If we assume that only particle 1 is near-critical in Eq. (3.4), we obtain

$$
\frac{E_{\mathrm{cm}}}{2 m_{0}} \approx \sqrt{\frac{4+l_{H 1}^{2}}{16} \frac{l_{H 2}-l_{2}}{l_{H 1}-l_{1}}}
$$

For $e_{1}=e_{2}=e$, we denote $l_{H 1}=l_{H 2}=l_{H}$ and Eq. (3.4) reduces to

$$
\frac{E_{\mathrm{cm}}}{2 m_{0}}=\sqrt{1+\frac{\left(l_{1}-l_{2}\right)^{2}\left(4+l_{H}^{2}\right)}{16\left(l_{H}-l_{1}\right)\left(l_{H}-l_{2}\right)}},
$$

which reproduces the corresponding formula in [5]. When we set $e_{1}=e_{2}=1, l_{1}=l_{R}$ and $l_{2}=l_{L}$ in Eq. (3.7), we obtain

$$
\frac{E_{\mathrm{cm}}}{2 m_{0}}=\frac{1}{\sqrt[4]{1-a_{*}^{2}}} \sqrt{\frac{\left(1-a_{*}^{2}\right)+\left(1+\sqrt{1+a_{*}}+\sqrt{1-a_{*}}\right)^{2}}{1+\sqrt{1-a_{*}^{2}}}} .
$$


This reproduces the corresponding formula in [5]. This provides an upper bound for the collision of two marginally bound particles. For $a_{*}=0.998, E_{\mathrm{cm}} /\left(2 m_{0}\right) \simeq 9.49$ for this collision. In the limit $a_{*} \rightarrow 1$, we have

$$
\frac{E_{\mathrm{cm}}}{2 m_{0}} \approx \frac{1+\sqrt{2}}{\sqrt[4]{1-a_{*}^{2}}} \simeq \frac{2.41}{\sqrt[4]{1-a_{*}^{2}}}
$$

which are given in $[3] 5]$.

If $e_{1}=1$ and $l_{1}=l_{R}$ for particle 1 and particle 2 takes a subcritical orbit, we obtain

$$
\frac{E_{\mathrm{cm}}}{2 m_{0}} \approx \frac{1}{\sqrt{2} \sqrt{2-\sqrt{2}}} \frac{\sqrt{2 e_{2}-l_{2}}}{\sqrt[4]{1-a_{*}^{2}}}
$$

in the near-extremal limit. Although the numerical factor depends on the choice of $e_{2}$ and $l_{2}$, the proportionality to $\left(1-a_{*}^{2}\right)^{-1 / 4}$ does not change as long as $e_{1}=1$ and $l_{1}=l_{R}$.

\section{B. Near-horizon collision around an extremal Kerr black hole}

For the extremal Kerr black hole $a_{*}^{2}=1$, the effective potential is given by

$$
g(y)=-2(l-e)^{2} y^{3}+\left(l^{2}-e^{2}+1\right) y^{2}-2 y-\left(e^{2}-1\right) .
$$

The double root $y=y_{H}=1$ of $D(y)$ gives an event horizon. We should note that the region outside the horizon, $r>M$, is transformed to $0<y<1$.

From Eqs. (3.1) and (3.2), for the critical orbit $l=l_{H}=2 e$, we find $g\left(y_{H}\right)=g^{\prime}\left(y_{H}\right)=0$ and hence $y=y_{H}$ is a stationary point of the effective potential. In fact, for the critical orbit, we have

$$
g(y)=-\left(3 e^{2}-1\right)(1-y)^{2}+2 e^{2}(1-y)^{3} .
$$

Thus, there exists a critical orbit in the vicinity of the horizon if and only if $3 e^{2}>1$ and then the effective potential takes a maximum which is zero at $y=y_{H}=1$. From this fact, one might infer an unstable circular orbit for a massive particle at $y=y_{H}$, i.e., on the horizon which is a null hypersurface. This apparent paradox is resolved in Sec. IIIC. On the other hand, the maximal point on the horizon implies the existence of an orbit for a massive particle with $l=l_{H}$ which asymptotes the horizon. In fact, from Eq. (3.12) we have

$$
\dot{r}=u^{r}=-\sqrt{3 e^{2}-1}(1-y) \sqrt{1-\frac{2 e^{2}}{3 e^{2}-1}(1-y)} .
$$


and this can be integrated to give

$$
\ln |r-M| \approx-\sqrt{3 e^{2}-1} \frac{\tau}{M}+\text { const }
$$

near the horizon. Thus, the critical particle approaches the horizon as $\tau \rightarrow \infty$, as shown in [4, 5]. For the subcritical orbit $l<2 e$, since

$$
-g(y)=[(2 e-l)-2(e-l)(1-y)]^{2}-[1-(e-l)(3 e-l)](1-y)^{2}-2(e-l)^{2}(1-y)^{3},
$$

we have a different behavior of $u^{r}$ as

$\dot{r}=u^{r}=-[(2 e-l)-2(e-l)(1-y)] \sqrt{1-\frac{[1-(e-l)(3 e-l)]+2(e-l)^{2}(1-y)}{[(2 e-l)-2(e-l)(1-y)]^{2}}(1-y)^{2}}$.

The proper time for the subcritical particle to reach the horizon is inversely proportional to $(2 e-l)$ because Eq. (3.3) is still valid even in the extremal Kerr case.

If particle 1 takes a critical orbit but particle 2 takes a subcritical orbit, the CM energy is given by the near-horizon limit of Eq. (2.10) as

$$
\frac{E_{\mathrm{cm}}}{2 m_{0}} \approx \sqrt{\frac{\left(2 e_{2}-l_{2}\right)\left(2 e_{1}-\sqrt{3 e_{1}^{2}-1}\right)}{2(1-y)}}
$$

where we have used Eq. (3.13). For the special case $e_{1}=e_{2}=e$, Eq. (3.14) reproduces the corresponding formula in [5].

If both particles take subcritical orbits, the fraction on the right-hand side of Eq. (2.10) is bounded because both the numerator $F-G$ and the denominator $D$ have a second-order zero at the horizon $y=y_{H}=1$. Estimating the terms of $O\left((1-y)^{2}\right)$ in $F-G$ and $D$ by Taylor series expansion, we obtain

$$
\frac{E_{\mathrm{cm}}}{2 m_{0}}=\sqrt{\frac{1}{2}\left[1-e_{1} e_{2}+\frac{2 e_{2}-l_{2}}{2 e_{1}-l_{1}} \frac{1+e_{1}^{2}}{2}+\frac{2 e_{1}-l_{1}}{2 e_{2}-l_{2}} \frac{1+e_{2}^{2}}{2}\right]} .
$$

More systematically, we can take the following approach. For the extremal case, the numerator $F-G$ and the denominator $D$ both must have a second-order zero at $y=y_{H}$. Using l'Hospital's rule twice, we obtain

$$
\frac{E_{\mathrm{cm}}^{2}}{2 m_{0}^{2}}=1-e_{1} e_{2}+\lim _{y \rightarrow y_{H}} \frac{F^{\prime \prime}-G^{\prime \prime}}{D^{\prime \prime}}
$$


where the second-order derivatives are given by

$$
\begin{aligned}
F^{\prime \prime}(y) & =4\left[a_{*}^{2}(1+3 y)\right] e_{1} e_{2}-12 a_{*} y\left(e_{1} l_{2}+l_{1} e_{2}\right)-2(1-6 y) l_{1} l_{2}, \\
g_{i}^{\prime \prime}(y) & =-12\left(l_{i}-a_{*} e_{i}\right)^{2} y+2\left[l_{i}^{2}-a_{*}^{2}\left(e_{i}^{2}-1\right)\right], \\
D^{\prime \prime}(y) & =2 a_{*}^{2}, \\
G^{\prime \prime} & =G\left[\frac{1}{2}\left(\frac{g_{1}^{\prime \prime}}{g_{1}}+\frac{g_{2}^{\prime \prime}}{g_{2}}\right)-\frac{1}{4}\left(\frac{g_{1}^{\prime}}{g_{1}}-\frac{g_{2}^{\prime}}{g_{2}}\right)^{2}\right]
\end{aligned}
$$

and $a_{*}=1$ for the extremal case. It is found that this approach also yields Eq. (3.15).

In the course of derivation, it is not so obvious whether the formula for the subextremal Kerr black hole given by Eq. (3.4) reproduces the formula (3.15) for the extremal case if we take the near-extremal limit $a_{*} \rightarrow 1$ in the former. In fact, it is not difficult to see that this is the case by putting $l_{H i}=2 e_{i}$ in Eq. (3.4). Therefore, the general formula (3.4) or (3.5),

which has been derived for the subextremal case, is applicable in both the subextremal and extremal cases.

We can confirm that for the special case $e_{1}=e_{2}=e$, Eq. (3.15) reduces to

$$
\frac{E_{\mathrm{cm}}}{2 m_{0}}=\sqrt{1+\frac{1+e^{2}}{4} \frac{\left(l_{1}-l_{2}\right)^{2}}{\left(2 e-l_{1}\right)\left(2 e-l_{2}\right)}},
$$

which coincides with the corresponding formula in [5]. Moreover, Eq. (3.15) reduces to

$$
\frac{E_{\mathrm{cm}}}{2 m_{0}}=\sqrt{\frac{1}{2}\left(\frac{2-l_{1}}{2-l_{2}}+\frac{2-l_{2}}{2-l_{1}}\right)},
$$

for the special case $e_{1}=e_{2}=1$, which reproduces the formula discovered by BSW [1] .

\section{The circular timelike orbit on the extremal Kerr black hole horizon is fake}

As we have seen in Sec. IIB , in the extremal Kerr case, $r=r_{H}$ is a zero and maximal point of the effective potential. From this fact, one might infer that an unstable circular orbit for a massive particle is possible at $r=r_{H}$. However, we will show that this is not real.

We should note that the Boyer-Lindquist coordinate system has a coordinate singularity at $r=r_{H}$. To avoid the complication due to the coordinate singularity, we move to the ingoing Kerr coordinates [21]:

$$
d v=d t+\left(r^{2}+a^{2}\right) \frac{d r}{\Delta}, \quad d \varphi=d \phi+a \frac{d r}{\Delta} .
$$


The line element then can be written as

$$
\begin{aligned}
d s^{2}= & -\left(1-\frac{2 M r}{\rho^{2}}\right) d v^{2}+2 d v d r+\rho^{2} d \theta^{2}+\frac{\left[\left(r^{2}+a^{2}\right)^{2}-a^{2} \Delta \sin ^{2} \theta\right] \sin ^{2} \theta}{\rho^{2}} d \varphi^{2} \\
& -2 a \sin ^{2} \theta d \varphi d r-\frac{4 a M r}{\rho^{2}} \sin ^{2} \theta d \varphi d v .
\end{aligned}
$$

The Killing vectors are given by

$$
\xi^{a}=\left(\frac{\partial}{\partial v}\right)^{a}, \quad \psi^{a}=\left(\frac{\partial}{\partial \varphi}\right)^{a} .
$$

On the equatorial plane $\theta=\pi / 2$, the line element in the extremal Kerr spacetime is given by

$$
d s^{2}=-\left(1-\frac{2 M}{r}\right) d v^{2}+2 d v d r+\left(r^{2}+M^{2}+\frac{2 M^{3}}{r}\right) d \varphi^{2}-2 M d \varphi d r-\frac{4 M^{2}}{r} d \varphi d v .
$$

The conserved quantities are given by

$$
\begin{aligned}
& e=-g_{a b} \xi^{a} u^{b}=\left(1-\frac{2 M}{r}\right) \dot{v}-\dot{r}+\frac{2 M^{2}}{r} \dot{\varphi}, \\
& L=g_{a b} \psi^{a} u^{b}=\left(r^{2}+M^{2}+\frac{2 M^{3}}{r}\right) \dot{\varphi}-M \dot{r}-\frac{2 M^{2}}{r} \dot{v} .
\end{aligned}
$$

Putting $r=r_{H}=M$ in Eqs. (3.17) and (3.18), we have

$$
\begin{aligned}
e & =-\dot{v}-\dot{r}+2 M \dot{\varphi} \\
L & =-2 M \dot{v}-M \dot{r}+4 M^{2} \dot{\varphi} .
\end{aligned}
$$

The norm of $u^{a}$ can be written at $r=M$ as

$$
u^{a} u_{a}=\dot{v}^{2}+2 \dot{r} \dot{v}+4 M^{2} \dot{\varphi}^{2}-2 M \dot{\varphi} \dot{r}-4 M \dot{\varphi} \dot{v}
$$

If we assume that the particle remains on the horizon $r=M$, we have $\dot{r}=0$ and we may conclude $L / e=2 M$ or $e=L=0$ from Eqs. (3.19) and (3.20). On the other hand, one cannot solve Eqs. (3.19) and (3.20) for $\dot{v}$ and $\dot{\varphi}$ separately in terms of $e$ and $L$ because of the degeneracy and hence cannot obtain Eqs. (2.6) and (2.7). In other words, Eqs. (2.6) and (2.7) do not make sense in the present case. Instead, we obtain from Eqs. (3.19) and (3.21)

$$
u^{a} u_{a}=e^{2}
$$

Since $u^{a} u_{a} \leq 0$ for causal geodesics, this means that a causal geodesic can remain on the horizon only if it is a null geodesic with $e=L=0$. Thus, the timelike circular orbit on 
the horizon, which might be inferred from the stationary point of the effective potential at $r=r_{H}$, is fake. Note also that the angular velocity of this null geodesic which remains on the horizon is given by

$$
\frac{d \varphi}{d v}=\frac{\dot{\varphi}}{\dot{v}}=\frac{1}{2 M}=\Omega_{H},
$$

indicating that this null geodesic is a generator of the event horizon.

This is of course entirely consistent with the following general argument. Any timelike curve cannot remain on an event horizon because the event horizon is normal to the Killing vector $\chi^{a}$, which is null on the horizon, and hence the tangent space at a point on the horizon is spanned by $\chi^{a}$ and two spacelike vectors $e_{(1)}^{a}$ and $e_{(2)}^{a}$ which are orthogonal to $\chi^{a}$. Any linear combination of the null vector $\chi^{a}$ and the spacelike vectors $e_{(1)}^{a}$ and $e_{(2)}$ is either null or spacelike.

\section{NEAR-HORIZON COLLISION OF A PARTICLE PLUNGING FROM THE ISCO}

As is emphasized in [5] and in Sec. [IA, the upper bound (3.9) applies only for the particles which begin at rest at infinity and reach the horizon all the way from infinity along the geodesic. If a particle loses or gains its energy or angular momentum on the way to the horizon, this limit does not apply. In this respect, particles plunging from the ISCO are considered very natural as particles plunging into the horizon in an astrophysical context.

The ISCO in the Kerr spacetime is explicitly given by Bardeen, Press and Teukolsky [9]. The circular orbit on the equatorial plane in the Kerr metric is given by $V_{\text {eff }}(r)=V_{\text {eff }}^{\prime}(r)=0$, where the prime denotes the derivative with respect to the argument. The condition implies

$$
\begin{aligned}
e & =\frac{r^{1 / 2}(r-2 M)+s a M^{1 / 2}}{r^{3 / 4}\left(r^{3 / 2}-3 M r^{1 / 2}+s 2 a M^{1 / 2}\right)^{1 / 2}}, \\
L & =s \frac{M^{1 / 2}\left(r^{2}+a^{2}-s 2 M^{1 / 2} a r^{1 / 2}\right)}{r^{3 / 4}\left(r^{3 / 2}-3 M r^{1 / 2}+s 2 a M^{1 / 2}\right)^{1 / 2}},
\end{aligned}
$$

where we have assumed $0 \leq a<M$ and $s=1$ and -1 correspond to the prograde and retrograde orbits, respectively. The ISCO is determined by the condition $d e / d r=d L / d r=$ 0 . The radius of the ISCO is then given by

$$
\begin{aligned}
\frac{r_{\mathrm{ISCO}}}{M} & =3+Z_{2}-s\left[\left(3-Z_{1}\right)\left(3+Z_{1}+2 Z_{2}\right)\right]^{1 / 2}, \\
Z_{1} & =1+\left(1-a_{*}^{2}\right)^{1 / 3}\left[\left(1-a_{*}\right)^{1 / 3}+\left(1+a_{*}\right)^{1 / 3}\right], \quad Z_{2}=\left(3 a_{*}^{2}+Z_{1}^{2}\right)^{1 / 2},
\end{aligned}
$$


where $0 \leq a_{*}<1$. The energy and angular momentum of the particle at the ISCO are calculated by substituting Eqs. (4.3) and (4.4) for $r=r_{\mathrm{ISCO}}$ into Eqs. (4.1) and (4.2).

To see the behavior in the near-extremal limit $a_{*} \rightarrow 1$, we put $a_{*}=1-\epsilon$ and expand the above obtained expression in terms of $\epsilon$. From Eqs. (4.4), we obtain

$$
Z_{1}=1+2^{2 / 3} \epsilon^{1 / 3}+2^{1 / 3} \epsilon^{2 / 3}+O(\epsilon), \quad Z_{2}=2+\frac{1}{2} 2^{2 / 3} \epsilon^{1 / 3}+\frac{7}{8} 2^{1 / 3} \epsilon^{2 / 3}+O(\epsilon) .
$$

Then, using Eqs. (4.1), (4.2) and (4.3), we obtain

$$
\begin{aligned}
\frac{r_{\mathrm{ISCO}}}{M} & =1+2^{2 / 3} \epsilon^{1 / 3}+\frac{7}{4} 2^{1 / 3} \epsilon^{2 / 3}+O(\epsilon), \\
e & =\frac{\sqrt{3}}{3}+\frac{2^{2 / 3}}{3} \sqrt{3} \epsilon^{1 / 3}-\frac{5}{12} 2^{1 / 3} \sqrt{3} \epsilon^{2 / 3}+O(\epsilon), \\
l & =\frac{2}{3} \sqrt{3}+\frac{2}{3} 2^{2 / 3} \sqrt{3} \epsilon^{1 / 3}+\frac{1}{6} 2^{1 / 3} \sqrt{3} \epsilon^{2 / 3}+O(\epsilon),
\end{aligned}
$$

and hence

$$
\frac{l}{e}=2+3 \cdot 2^{1 / 3} \epsilon^{2 / 3}+O(\epsilon)
$$

for the prograde orbit $(s=1)$. For the retrograde orbit $(s=-1)$, we obtain

$$
\begin{aligned}
\frac{r_{\mathrm{ISCO}}}{M} & =9+O(\epsilon), \\
e & =\frac{5}{9} \sqrt{3}+O(\epsilon), \\
l & =-\frac{22}{9} \sqrt{3}+O(\epsilon),
\end{aligned}
$$

and hence

$$
\frac{l}{e}=-\frac{22}{5}+O(\epsilon)
$$

On the other hand, $l_{H}$ can be written as

$$
\frac{l_{H}}{e}=\frac{2 a_{*}}{1-\sqrt{1-a_{*}^{2}}}=2+2 \sqrt{2} \epsilon^{1 / 2}+O(\epsilon) .
$$

Therefore, the prograde ISCO particle has the angular momentum which coincides with the critical value $l_{H}$ in the near-extremal limit.

We should note that for the marginally bound particle with $l=l_{R}$ we have $l / e=l_{R}=$ $2+2 \epsilon^{1 / 2}$. Therefore, $\left(l_{H}-l\right) / e \approx 2 \sqrt{2} \epsilon^{1 / 2}$ for the prograde ISCO, while $\left(l_{H}-l\right) / e \approx$ $2(\sqrt{2}-1) \epsilon^{1 / 2}$ for a marginally bound particle with $l=l_{R}$. Since all other factors are nonzero finite, a particle which plunges from the prograde ISCO collides with a generic particle with $E_{\mathrm{cm}} \propto \epsilon^{-1 / 4}$, as a marginally bound particle with $l=l_{R}$ does. It also follows 
that if a particle plunging from the prograde ISCO collides with a marginally bound particle with $l=l_{R}$, the CM energy is bounded even in the near-extremal limit $a_{*} \rightarrow 1$.

Using Eq. (3.6) with Eqs. (4.5)-(4.7), we can easily estimate the CM energy near the horizon for the near-extremal Kerr black hole. If particle 1 is a particle plunging from the prograde ISCO and particle 2 takes a subcritical orbit, we obtain

$$
\frac{E_{\mathrm{cm}}}{2 m_{0}} \approx \frac{1}{2^{1 / 2} 3^{1 / 4}} \frac{\sqrt{2 e_{2}-l_{2}}}{\sqrt[4]{1-a_{*}^{2}}}
$$

Thus, the CM energy can be unboundedly high in the limit $a_{*} \rightarrow 1$. Since the dependence $\left(1-a_{*}\right)^{-1 / 4}$ is common to the upper bound (3.9) for marginally bound particles, we can conclude that the BSW effect occurs for a particle plunging from the prograde ISCO and in this case the fine-tuning of the energy and the angular momentum is naturally realized in the standard accretion disks with electromagnetic radiation or in inspiralling binaries with gravitational wave radiation. The ratio of $E_{\mathrm{cm}}$ for the ISCO particle to that for the marginally bound particle with $l=l_{R}$ is given by $\sqrt{2-\sqrt{2}} / 3^{1 / 4} \simeq 0.582$ in the near-extremal limit.

In the following, we consider the near-horizon collisions of a particle plunging from the prograde ISCO with (a) a marginally bound particle with $l=l_{L}$, (b) a particle plunging from the retrograde ISCO, and (c) a marginally bound particle with $l=0$. In the near-extremal limit $a_{*} \rightarrow 1$, we obtain

$$
\frac{E_{\mathrm{cm}}}{2 m_{0}} \approx \frac{\alpha}{\sqrt[4]{1-a_{*}^{2}}}
$$

where the numerical factor $\alpha=\sqrt{2 e_{2}-l_{2}} /\left(2^{1 / 2} 3^{1 / 4}\right)$ is calculated to be $\sqrt{(2+\sqrt{2}) / \sqrt{3}} \simeq$ $1.40,4 / 3 \simeq 1.33$ and $1 / \sqrt[4]{3} \simeq 0.760$ for cases (a), (b), and (c), respectively.

For the general values of $a_{*}$ in $0 \leq a_{*}<1$, we can calculate the CM energy using Eq. (3.4) with Eqs. (4.1)-(4.4). The result is summarized in Fig. 1, where $E_{\mathrm{cm}} /\left(2 m_{0}\right)$ is multiplied by $\sqrt[4]{1-a_{*}^{2}}$ for clarity. In this figure, the solid, dashed, and dotted curves denote cases (a), (b), and (c), respectively. The CM energy for two particles, either of which is a particle plunging from the prograde ISCO is always below the upper bound (3.8) for marginally bound particles. We should note that the dependence of $\left(E_{\mathrm{cm}} /\left(2 m_{0}\right)\right)\left(1-a_{*}^{2}\right)^{1 / 4}$ on $a_{*}$ in $0 \leq a_{*}<1$ is very weak for cases (a), (b), and (c) and hence the empirical formula $E_{\mathrm{cm}} /\left(2 m_{0}\right) \sim 1 / \sqrt[4]{1-a_{*}^{2}}$ is a very good approximation within a factor of 2 or so for all values of $a_{*}$ in $0 \leq a_{*}<1$. Thus, this formula provides the typical value for the CM energy 
for the near-horizon collision of a particle which plunges from the prograde ISCO with a generic subcritical particle. For the near-maximal rotation, the maximum value for the $\mathrm{CM}$ energy coincides with the upper bound for a marginally bound particle within a factor of 2 .

If we use Thorne's bound $a_{*}=0.998$ for the spin parameter, $E_{\mathrm{cm}} /\left(2 m_{0}\right)$ is calculated to be $6.95,6.61$, and 3.86 for cases (a), (b), and (c), respectively. This means that a highly relativistic collision can naturally occur near the horizon of a rapidly rotating black hole in an astrophysical context. Note that with highly relativistic collision we here mean systems where the CM energy is much larger than the rest mass.

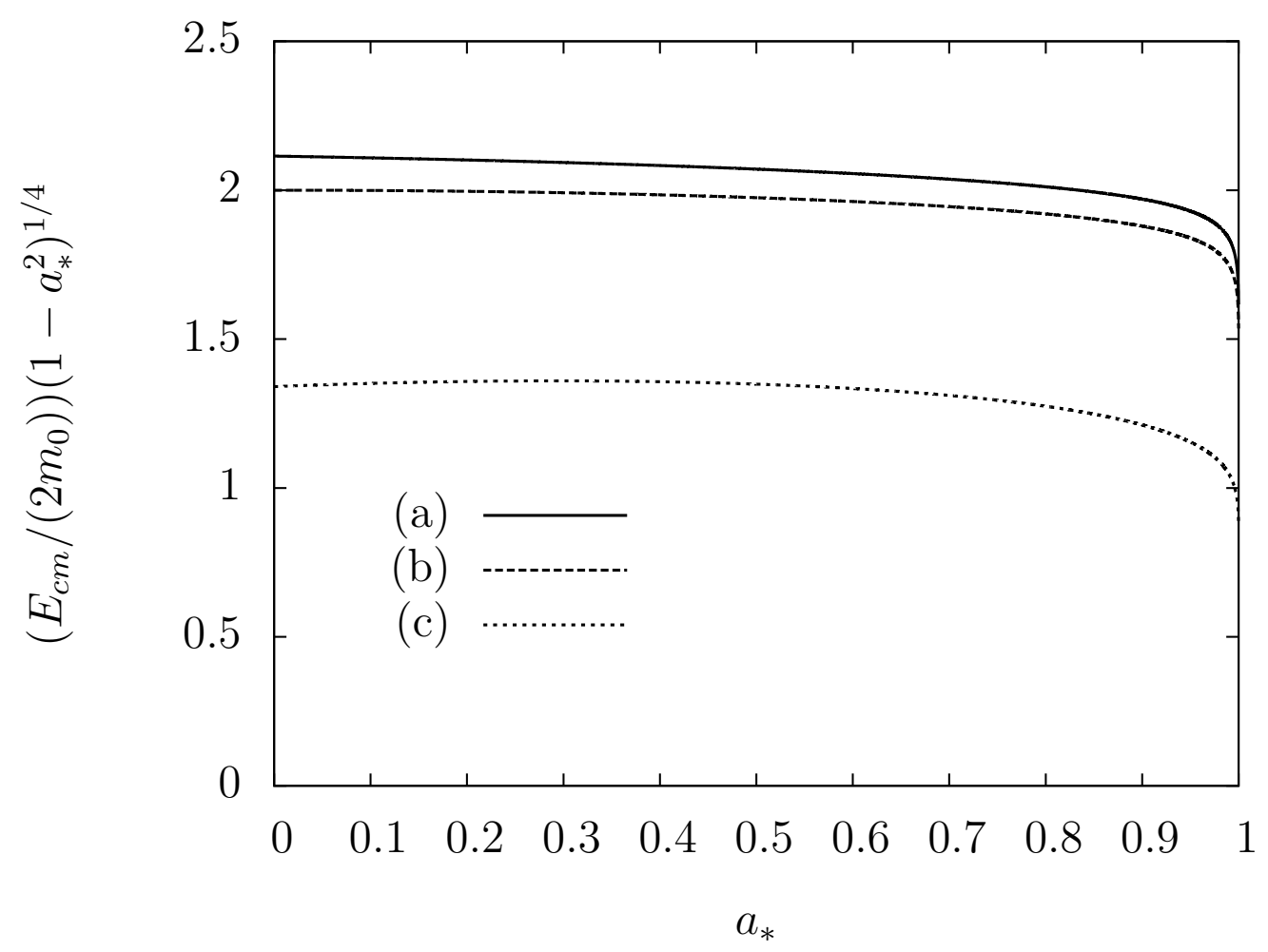

FIG. 1: The CM energy $E_{\mathrm{cm}}$ for the near-horizon collision. The solid, dashed, and dotted curves denote the collisions of a particle plunging from the prograde ISCO with (a) a marginally bound particle with $l=l_{L}$, (b) a particle plunging from the retrograde ISCO, and (c) a marginally bound particle with $l=0$, respectively. For clarity, $E_{\mathrm{cm}}$ is multiplied by $\sqrt[4]{1-a_{*}^{2}}$ in the vertical axis.

\section{PARTICLE COLLISION OF A PARTICLE ORBITING THE ISCO}

In this section, we deviate somewhat from the original idea of BSW [1]. We consider the situation where a particle orbiting the ISCO collides with another particle on the ISCO 
instead of a near-horizon collision. In this case, we cannot take the near-horizon limit beforehand. Although we do not expect a compact expression for the general case, we can obtain a simple formula for the near-extremal limit. Using Eq. (2.10) with Eqs. (2.11)-(2.14) and (4.5)-(4.7) and $y=M / r_{\mathrm{ISCO}}$, we can estimate the CM energy for the near-extremal Kerr black hole. If particle 1 is a particle orbiting the prograde ISCO and particle 2 takes a subcritical orbit, we obtain

$$
\frac{E_{\mathrm{cm}}}{2 m_{0}} \approx \frac{1}{2^{1 / 6} 3^{1 / 4}} \frac{\sqrt{2 e_{2}-l_{2}}}{\sqrt[6]{1-a_{*}^{2}}} .
$$

It is quite intriguing that the dependence $\left(1-a_{*}^{2}\right)^{-1 / 6}$ on the spin parameter here is quite different from that for the near-horizon collision $\left(1-a_{*}^{2}\right)^{-1 / 4}$. The CM energy can be arbitrarily high in the near-extremal limit $a_{*} \rightarrow 1$. However, this needs to be distinguished from the BSW effect for the near-horizon collision of plunging particles.

As in the near-horizon case, we consider the "on-ISCO" collisions of a particle orbiting the prograde ISCO with (a) a marginally bound particle with $l=l_{L}$, (b) a particle plunging from the retrograde ISCO, and (c) a marginally bound particle with $l=0$. In the nearextremal limit $a_{*} \rightarrow 1$, we obtain

$$
\frac{E_{\mathrm{cm}}}{2 m_{0}} \approx \frac{\beta}{\sqrt[6]{1-a_{*}^{2}}}
$$

where the numerical factor $\beta=\sqrt{2 e_{2}-l_{2}} /\left(2^{1 / 6} 3^{1 / 4}\right)$ is calculated to be $\sqrt{2(2+\sqrt{2})} /\left(2^{1 / 6} 3^{1 / 4}\right) \simeq 1.77,4 \cdot 2^{1 / 3} / 3 \simeq 1.68$, and $\sqrt{2} /\left(2^{1 / 6} 3^{1 / 4}\right) \simeq 0.957$ for cases (a), (b), and (c), respectively.

For the general values of $a_{*}$ in $0 \leq a_{*}<1$, we can calculate the CM energy using Eq. (2.10) with Eqs. (2.11)-(2.14) and (4.1)-(4.4), $r=r_{\text {ISCO }}$ and $y=M / r_{\text {ISCO }}$. The result is summarized in Fig. 2, where $E_{\mathrm{cm}} /\left(2 m_{0}\right)$ is multiplied by $\sqrt[6]{1-a_{*}^{2}}$ for clarity. In this figure, the solid, dashed, and dotted curves denote cases (a), (b), and (c), respectively. The CM energy for two particles, either of which is a particle orbiting the prograde ISCO, is always below the upper bound (3.8) for marginally bound particles. We should note that the dependence of $\left(E_{\mathrm{cm}} /\left(2 m_{0}\right)\right)\left(1-a_{*}^{2}\right)^{1 / 6}$ on $a_{*}$ in $0 \leq a_{*}<1$ is very weak and hence the empirical formula $E_{\mathrm{cm}} /\left(2 m_{0}\right) \sim 1 / \sqrt[6]{1-a_{*}^{2}}$ is a very good approximation within a factor of 2 for all values of $a_{*}$ in $0 \leq a_{*}<1$. Thus, this formula provides the typical value for the CM energy for the on-ISCO collision of a particle which orbits the prograde ISCO with a generic subcritical particle. It should be noted that the CM energy for the on-ISCO collision is 
always smaller than that for the near-horizon collision of a particle plunging from the ISCO in the near-extremal limit because of the different dependence on $a_{*}$.

If we use Thorne's bound $a_{*}=0.998$ for the spin parameter, $E_{\mathrm{cm}} /\left(2 m_{0}\right)$ is calculated to be $4.11,3.91$, and 2.43 for cases (a), (b), and (c), respectively. This means that a highly or moderately relativistic collision can naturally occur on the ISCO around a rapidly rotating black hole in an astrophysical context.

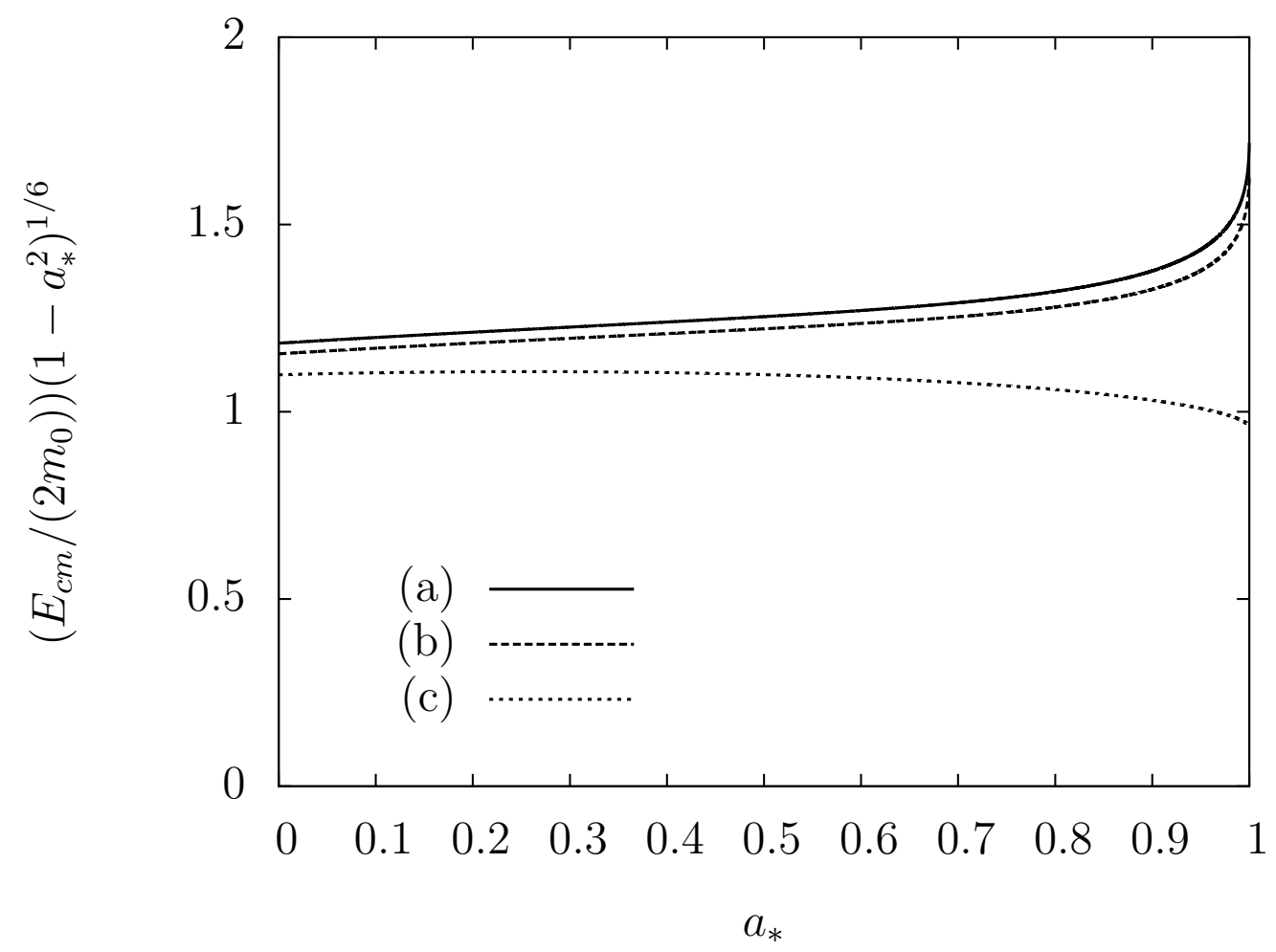

FIG. 2: The CM energy $E_{\mathrm{cm}}$ for the on-ISCO collision. The solid, dashed, and dotted curves denote the collisions of a particle orbiting the prograde ISCO with (a) a marginally bound particle with $l=l_{L}$, (b) a particle plunging from the retrograde ISCO, and (c) a marginally bound particle with $l=0$, respectively. For clarity, $E_{\mathrm{cm}}$ is multiplied by $\sqrt[6]{1-a_{*}^{2}}$ in the vertical axis.

\section{CONCLUSION AND DISCUSSION}

We have investigated particle collisions near the horizon and on the ISCO around a Kerr black hole. We have derived a general explicit formula for the CM energy near the horizon in terms of the energies and the angular momenta of colliding two particles on the equatorial plane. We have confirmed that the obtained formula includes known formulas as its special 
cases. We have explicitly shown that although the effective potential around a maximally rotating Kerr black hole has a zero and maximal point on the horizon, it does not correspond to a real circular orbit. Then, we have studied the near-horizon collision of particles, either of which plunges from the ISCO. We have shown that the BSW effect occurs for such a collision in the near-maximal rotation limit and that the maximum value for the CM energy is the same within a factor of 2 as the upper bound for the marginally bound particles for which the angular momentum must be fine-tuned. We have also investigated the collision of a particle orbiting the ISCO with another generic particle on the ISCO and found that it is also the case that one can obtain an arbitrarily high CM energy in the near-maximal rotation limit, although this energy is smaller than the value for the near-horizon collision in this limit. The result implies that the BSW effect, which was originally proposed for the marginally bound particles with the fine-tuned angular momentum, is astrophysically relevant since the fine-tuning is naturally realized for ISCO particles in the standard accretion disks and extreme mass-ratio inspirals. Although the CM energy is bounded if the spin parameter of the black hole is bounded in an astrophysical context, the collision can still be highly or moderately relativistic near the horizon and on the ISCO around a rapidly rotating black hole.

The present result naively suggests the following scenario. A highly or moderately relativistic collision often occurs near the horizon of a rapidly rotating black hole in the context of the accretion disks and the extreme mass-ratio binaries. For the standard accretion disk, gamma rays with energy of several GeVs can be produced inside and around the inner edge of the disk, if the CM energies of protons and ions collisions are eventually converted to photons. These photons can have much higher energy than usual thermal photons.

What is more intriguing is the high-velocity collision of compact objects around a supermassive or intermediate-mass black hole. Here, the compact objects will collide near the horizon or on the ISCO with a high "relativistic gamma factor" $E_{\mathrm{cm}} /\left(2 m_{0}\right)$. The result will strongly depend on the kinds of the compact objects and the value of the relativistic gamma factor. For example, if two neutron stars collide with a sufficiently high gamma, it will result in the gravitational collapse to a black hole (e.g. [22]). If two white dwarfs collide with a sufficiently high gamma, they might be smashed, destroyed, and scattered away because of the CM energy much greater than the binding energy of the white dwarfs. Thus, the collision of the compact objects around a rapidly rotating supermassive or intermediate-mass 
black hole provides a unique laboratory for the relativistic collision of black holes, neutron stars, and white dwarfs. The interaction of the compact object with the fluid or plasma near the horizon or on the ISCO might also be a striking phenomenon. The details of all these processes would not be so simple and should be investigated not only by analytical arguments but also by numerical simulations, including numerical relativity and general relativistic hydrodynamics.

Finally, we speculate that peculiar signals originating from the highly or moderately relativistic collision of particles, fluids and compact objects around a rapidly rotating black hole might be detected by the direct observation of black holes by means of electromagnetic and/or gravitational waves and/or neutrinos. For example, if two black holes collide with a sufficiently high gamma, a considerable fraction (as large as $14 \pm 3 \%$ for head-on collision [23] and $35 \pm 5 \%$ for zoom-whirl collision [24, 25]) of the CM energy can be radiated away through gravitational radiation. Of course, because of the strong redshift, we cannot immediately expect that the energetic radiation can directly reach us. However, the emission peculiar to such relativistic collisions will be redshifted and might still be observed in electromagnetic and/or gravitational waves and/or neutrinos. In this respect, the on-ISCO collision might be more advantageous to observation than the near-horizon collision. To investigate what signals would be observed from a highly or moderately relativistic collision, numerical simulations will be very powerful.

\section{Acknowledgments}

The authors thank U. Miyamoto, K. Nakao, R. Takahashi, K. Hioki, M. Saijo, and M. Shibata for fruitful discussion. The authors also thank the anonymous referee for helpful comments. T.H. was supported by a Grant-in-Aid for Scientific Research from the Ministry of Education, Culture, Sports, Science and Technology of Japan [Young Scientists (B) No. $21740190]$.

[1] Maximo Banados, Joseph Silk, and Stephen M. West, "Kerr Black Holes as Particle Accelerators to Arbitrarily High Energy", Phys. Rev. Lett. 103, 111102 (2009). 
[2] Roy P. Kerr, "Gravitational Field of a Spinning Mass as an Example of Algebraically Special Metrics", Phys. Rev. Lett. 11, 237 (1963).

[3] Emanuele Berti, Vitor Cardoso, Leonardo Gualtieri, Frans Pretorius, and Ulrich Sperhake, "Comment on 'Kerr Black Holes as Particle Accelerators to Arbitrarily High Energy' ", Phys. Rev. Lett. 103, 239001 (2009).

[4] Ted Jacobson and Thomas P. Sotiriou, "Spinning Black Holes as Particle Accelerators", Phys. Rev. Lett. 104, 021101 (2010).

[5] Andrey A. Grib and Yuri V. Pavlov, "On particle collisions near Kerr's black holes", arXiv: $1007.3222 \mathrm{v} 1$.

[6] Shao-Wen Wei, Yu-Xiao Liu, Heng Guo, and Chun-E Fu, "Charged Spinning Black Holes as Particle Accelerators", Phys. Rev. D82, 103005 (2010).

[7] Oleg B. Zaslavskii, "Acceleration of particles as universal property of rotating black holes", Phys. Rev. D82, 083004 (2010).

[8] Oleg B. Zaslavskii, "Acceleration of particles by nonrotating charged black holes", Pis'ma Zh. Eksp. Teor. Fiz. 92, 635 (2010) [JETP Letters 92, 571 (2010)].

[9] James M. Bardeen, William H. Press, and Saul A. Teukolsky, "Rotating Black Holes: Locally Nonrotating Frames, Energy Extraction, and Scalar Synchrotron Radiation", Astrophys. J. 178, 347 (1972).

[10] N. I. Shakura and R. A. Sunyaev, "Black Holes in Binary Systems. Observational Appearance", Astron. Astrophys. 24, 337 (1973).

[11] Don N. Page and Kip S. Thorne, "Disk-Accretion onto a Black Hole. I. Time Averaged Structure of Accretion Disk", Astrophys. J 191, 499 (1974).

[12] Rohta Takahashi, "Horizon-penetrating transonic accretion discs around rotating black holes", Mon. Not. Roy. Astron. Soc. 382, 567 (2007).

[13] Shoji Kato, Jun Fukue, and Shin Mineshige, "Black-Hole Accretion Discs, Towards a New Paradigm" (Kyoto University Press, Kyoto, 2008).

[14] Kip S. Thorne, "Disk-Accretion onto a Black Hole. II. Evolution of the Hole", Astrophys. J. 191, 507 (1974).

[15] LISA, "Laser interferometer space antenna: a cornerstone mission for the observation of gravitational waves System and Technology Study Report", ESA-SCI p 11 (2000).

[16] Naoki Seto, Seiji Kawamura, and Takashi Nakamura, "Possibility of Direct Measurement of 
the Acceleration of the Universe Using $0.1 \mathrm{~Hz}$ Band Laser Interferometer Gravitational Wave Antenna in Space", Phys. Rev. Lett. 87, 221103 (2001).

[17] Seiji Kawamura et al., "The Japanese space gravitational wave antenna: DECIGO", Class. Quantum Grav. 23, S125 (2006).

[18] E. S. Phinney et al. "The big bang observer: direct detection of gravitational waves from the birth of the Universe to the present NASA Mission Concept Study" (2004).

[19] Jeff Crowder and Neil J. Cornish, "Beyond LISA: Exploring future gravitational wave missions", Phys. Rev. D72, 083005 (2005).

[20] Robert M. Wald, "General Relativity", (University of Chicago Press, Chicago, 1984).

[21] Eric Poisson, "A Relativist's Toolkit: The Mathematics of Black-Hole Mechanics", (Cambridge University Press, Cambridge, 2004).

[22] Mark A. Miller, Wai-Mo Suen, and Malcolm Tobias, "The Shapiro conjecture: Prompt or delayed collapse in the head-on collision of neutron stars?", Phys. Rev. D63, 121501 (2001).

[23] Ulrich Sperhake, Vitor Cardoso, Frans Pretorius, Emanuele Berti, Jose A. Gonzalez, "The high-energy collision of two black holes", Phys. Rev. Lett. 101, 161101 (2008).

[24] Masaru Shibata, Hirotada Okawa, and Tetsuro Yamamoto, "High-velocity collision of two black holes", Phys. Rev. D78, 101501(R) (2008).

[25] Ulrich Sperhake, Vitor Cardoso, Frans Pretorius, Emanuele Berti, Tanja Hinderer, Nicolas Yunes, "Cross section, final spin and zoom-whirl behavior in high-energy black hole collisions", Phys. Rev. Lett. 103, 131102 (2009).

[26] The fluid will not take a quasicircular orbit around the ISCO in the radiatively inefficient accretion flows, including the advection-dominated accretion flows, as shown in e.g. [12]. See also [13] for the accretion disk models. 